\title{
Chronic Villitis
}

National Cancer Institute

\section{Source}

National Cancer Institute. Chronic Villitis. NCI Thesaurus. Code C112846.

T-cell immune response with diffuse perivillous fibrin deposition associated with intrauterine growth restriction, pre-term birth and recurrent fetal loss. 